\title{
Cleft Lip/Palate-Ectodermal Dysplasia Syndrome
}

National Cancer Institute

\section{Source}

National Cancer Institute. Cleft Lip/Palate-Ectodermal Dysplasia Syndrome. NCI

Thesaurus. Code C122656.

A very rare genetic disorder characterized by cleft lip and palate, sparse scalp hair, and partial syndactyly of the fingers and toes. 